\title{
Off-Shell ADT Conserved Quantities in Palatini Gravity
}

\author{
Hai-Feng Ding ${ }^{1, *}$ and Xiang-Hua Zhai ${ }^{1,+}$ \\ ${ }^{1}$ Division of Mathematical and Theoretical Physics, \\ Shanghai Normal University, 100 Guilin Road, Shanghai 200234, China
}

In this paper we generalize the off-shell Abbott-Deser-Tekin (ADT) conserved charge formalism to Palatini theory of gravity with torsion and non-metricity. Our construction is based on the coordinate formalism and the independent dynamic fields are the metric and the affine connection. For a general Palatini theory of gravity, which is diffeomorphism invariant up to a boundary term, we obtain the most general expression for off-shell ADT potential. As explicit examples, we derive the off-shell ADT potentials for Einstein-Hilbert action, the most general $L\left(g_{\mu \nu}, R_{\nu \alpha \mu}^{\lambda}, T_{\alpha \beta}^{\lambda}, Q_{\alpha \mu \nu}\right)$ theories and the teleparallel Palatini gravity.

PACS numbers:

*haifeng1116@qq.com

tzhaixh@shnu.edu.cn 


\section{INTRODUCTION}

Symmetries and conservation laws play important roles in modern physics. In general theories of gravity, the construction of conserved quantities is a complex procedure. In order to obtain an effective way for computing the conserved quantities, several approaches have been proposed.

In the well known ADM approach [1], the conserved charges could be calculated for asymptotically flat spacetime at the asymptotic infinity. The extension to asymptotic (anti-)de sitter geometry has been given by Abbott-Deser-Tekin (ADT) [2 6] in a covariant manner. Based on the Noether's theorem, the covariant phase space method (CPSM) was proposed by Wald and Iyer [7-9]. In the framework of CPSM, a convenient method was developed for calculating conserved charges associated with 'exact symmetry' of black hole solutions [10 12], which is called solution phase space method (SPSM). To define the quasilocal energy, a covariant Hamiltonian method was developed by Chen, Nester and Tung [13 16]. Along another line, general theory of conserved charges based on the cohomology principles was developed by Barnich-Brandt-Compère (BBC) [17-20]. Recently, an off-shell ADT formalism was proposed by Bouchareb, Clément [21] and Kim-Kulkami-Yi in [22], and subsequently was generalized to including the matter fields [23, 24].

Each of these methods has its own merits and demerits, and most of these works were confined to pure Riemannian geometrical framework of gravity theories. Extentions to nonRiemannian geometries were studied in [25-29], and recently in [30 35]. The main property of non-Riemannian geometries is that the torsion and the non-metricity are included. The most general theory in non-Riemannian geometries is the Palatini gravity, which is called the Metric-Affine-Gravity (MAG) [25, 36] when the matter fields are included. Usually, the MAG is expressed in terms of two formalisms. The first one is written in the language of exterior differential forms, and the independent dynamic fields are the co-frame $e^{a}$ and the linear connection $\omega_{b}^{a}$, thus it is also named the frame-vielbein formalism. The second one is the coordinate formalism (namely, the usual tensoral language), and the independent dynamic fields are the metric $g_{\mu \nu}$ and the affine connection $\Gamma_{\mu \nu}^{\lambda}$.

To our knowledge, till now most of the constructions of conserved quantities in the nonRiemannian geometrical framework are in the language of exterior differential forms and are on shell, i.e. the equations of motion (EOM) are used. On the other hand, as mentioned 
in [32], when torsion is present, the derivations of the off-shell conserved quantities are complicated. The main reason is that the diffeomorphism variations of the metric and the affine connection cannot be written in terms of derivatives of the diffeomorphism vector fields. We will attempt to solve this problem by using the off-shell ADT formalism. In this paper, we will generalize the off-shell ADT formalism to the Palatini theory of gravity with torsion and non-metricity in the coordinate formalism.

The paper is organized as follows. In Sect. 2, some basic formulas in Palatini theory are presented for our subsequent derivation. In Sect. 3, we generalize the off-shell ADT formalism to Palatini theory with torsion and non-metricity. In Sect. 4, we derive the offshell ADT potentials for Einstein-Hilbert action, the most general $L\left(g_{\mu \nu}, R_{\nu \alpha \mu}^{\lambda}, T_{\alpha \beta}^{\lambda}, Q_{\alpha \mu \nu}\right)$ theories and the teleparallel Palatini gravity. In Sect. 5, the conserved charges are analyzed if the black hole solutions exist. The conclusions are given in the last section.

\section{PALATINI THEORIES}

We first fix our notations and conventions by briefly reviewing the basic geometrical objects in Palatini theory with torsion and non-metricity in the coordinate formalism [36], which will be used in our work.

We define the affine connection and the associated covariant derivative as

$$
\nabla_{\mu} u^{\nu}=\partial_{\mu} u^{\nu}+\Gamma_{\mu \sigma}^{\nu} u^{\sigma}
$$

where $u^{\nu}$ is an arbitrary vector field. In order to perform our subsequent derivation and make the expressions compact we define the transpose connection as $\bar{\Gamma}_{\mu \nu}^{\lambda}=\Gamma_{\nu \mu}^{\lambda}$, and the associated covariant derivative as $\bar{\nabla}_{\mu}$,

$$
\bar{\nabla}_{\mu} u^{\nu}=\partial_{\mu} u^{\nu}+\bar{\Gamma}_{\mu \sigma}^{\nu} u^{\sigma}=\partial_{\mu} u^{\nu}+\Gamma_{\sigma \mu}^{\nu} u^{\sigma}
$$

The basic geometrical objects in Palatini theory are torsion, non-metricity and curvature, which are defined as follows

$$
\begin{aligned}
T_{\mu \nu}^{\lambda} & =-2 \Gamma_{[\mu \nu]}^{\lambda}, \\
Q_{\alpha \mu \nu} & =-\nabla_{\alpha} g_{\mu \nu}=-\partial_{\alpha} g_{\mu \nu}+\Gamma_{\alpha \mu}^{\sigma} g_{\sigma \nu}+\Gamma_{\alpha \nu}^{\sigma} g_{\mu \sigma}, \\
R_{\nu \alpha \beta}^{\mu} & =2 \partial_{[\alpha} \Gamma_{\beta] \nu}^{\mu}+2 \Gamma_{[\alpha|\sigma|}^{\mu} \Gamma_{\beta] \nu}^{\sigma} .
\end{aligned}
$$


The commutators of covariant derivatives acting on a scalar $\phi$ and a vector $u^{\mu}$ are given by

$$
\begin{aligned}
{\left[\nabla_{\alpha}, \nabla_{\beta}\right] \phi } & =T_{\alpha \beta}^{\lambda} \nabla_{\lambda} \phi \\
{\left[\nabla_{\alpha}, \nabla_{\beta}\right] u^{\mu} } & =R_{\nu \alpha \beta}^{\mu} u^{\nu}+T_{\alpha \beta}^{\lambda} \nabla_{\lambda} u^{\mu} .
\end{aligned}
$$

It is easy to generalize the covariant derivatives and the commutators acting on arbitrary rank tensors. The contractions of the basic geometrical objects have the forms

$$
\begin{aligned}
R_{\nu \beta} & =R^{\mu}{ }_{\nu \mu \beta}, & R & =g^{\nu \beta} R_{\nu \beta}, \\
T_{\mu} & =T^{\lambda}{ }_{\mu \lambda}, & Q_{\alpha} & =g^{\mu \nu} Q_{\alpha \mu \nu}=Q_{\alpha \mu}{ }^{\mu}, \\
\check{R}^{\mu}{ }_{\beta} & =g^{\nu \alpha} R_{\nu \alpha \beta}^{\mu}, & \hat{R}_{\alpha \beta} & =R^{\mu}{ }_{\mu \alpha \beta}=\partial_{[\alpha} Q_{\beta]},
\end{aligned}
$$

where $R_{\nu \beta}$ is Ricci tensor, which is not symmetric in Palatini theory. The affine connection can be decomposed as

$$
\Gamma_{\mu \nu}^{\lambda}=\stackrel{\circ}{\Gamma}_{\mu \nu}^{\lambda}+N_{\mu \nu}^{\lambda},
$$

where $\stackrel{\circ}{\Gamma}^{\lambda}{ }_{\mu \nu}$ and $N^{\lambda}{ }_{\mu \nu}$ are Levi-Civita connection and the distorsion tensor, respectively, which are given by

$$
\begin{aligned}
\stackrel{\circ}{\Gamma}_{\mu \nu}^{\lambda} & =\frac{1}{2} g^{\lambda \alpha}\left(\partial_{\mu} g_{\nu \alpha}+\partial_{\nu} g_{\mu \alpha}-\partial_{\alpha} g_{\mu \nu}\right), \\
N_{\mu \nu}^{\lambda} & =K^{\lambda}{ }_{\mu \nu}+L_{\mu \nu}^{\lambda} \\
& =\frac{1}{2} g^{\lambda \alpha}\left(T_{\mu \nu \alpha}+T_{\nu \mu \alpha}-T_{\alpha \mu \nu}\right)+\frac{1}{2} g^{\lambda \alpha}\left(Q_{\mu \nu \alpha}+Q_{\nu \mu \alpha}-Q_{\alpha \mu \nu}\right),
\end{aligned}
$$

where $L^{\lambda}{ }_{\mu \nu}$ and $K^{\lambda}{ }_{\mu \nu}$ are called the disformation and the contorsion tensors, respectively.

Using the definition of the Riemann tensor and applying the Jacobi identity of covariant derivatives to scalars and vectors, one can obtain the Bianchi identities

$$
\begin{aligned}
R_{\nu(\alpha \beta)}^{\mu} & =0, \\
R_{[\alpha \beta \gamma]}^{\lambda}+\nabla_{[\alpha} T_{\beta \gamma]}^{\lambda}+T_{[\alpha \beta}^{\sigma} T_{\gamma] \sigma}^{\lambda} & =0 \\
\nabla_{[\alpha} R^{\sigma}{ }_{|\rho| \beta \gamma]}+T_{[\alpha \beta}^{\lambda} R_{|\rho| \gamma] \lambda}^{\sigma} & =0 .
\end{aligned}
$$

The variations of the basic geometrical objects have the following forms

$$
\begin{aligned}
\delta_{g} T_{\mu \nu}^{\lambda} & =0, & \delta_{\Gamma} T_{\alpha \beta}^{\lambda} & =-2 \delta^{[\mu}{ }_{\alpha} \delta_{\beta}^{\nu]} \delta \Gamma_{\mu \nu}^{\lambda}, \\
\delta_{g} R^{\mu}{ }_{\nu \alpha \beta} & =0, & \delta_{\Gamma} R^{\mu}{ }_{\nu \alpha \beta} & =\nabla_{\alpha} \delta \Gamma_{\beta \nu}^{\mu}-\nabla_{\beta} \delta \Gamma_{\alpha \nu}^{\mu}-T_{\alpha \beta}^{\lambda} \delta \Gamma_{\lambda \nu}^{\mu}, \\
\delta_{g} Q_{\rho \mu \nu} & =-\nabla_{\rho} \delta g_{\mu \nu}, & \delta_{\Gamma} Q_{\rho \alpha \beta} & =2 \delta^{\mu}{ }_{\rho} \delta^{\nu}{ }_{(\alpha} g_{\beta) \lambda} \delta \Gamma^{\lambda}{ }_{\mu \nu},
\end{aligned}
$$


where the torsion and the curvature tensors are independent of the metric.

The covariant derivatives of metric determinant and the non-trivial surface term are given by

$$
\begin{aligned}
\nabla_{\mu} \sqrt{-g} & =\partial_{\mu} \sqrt{-g}-\Gamma_{\mu \alpha}^{\alpha} \sqrt{-g}, \\
\nabla_{\mu}\left(\sqrt{-g} u^{\mu}\right) & =\partial_{\mu}\left(\sqrt{-g} u^{\mu}\right)+\sqrt{-g} T_{\mu} u^{\mu}, \\
\nabla_{[\mu} Q_{\nu] \alpha \beta} & =R_{(\alpha \beta) \mu \nu}+\frac{1}{2} T_{\mu \nu}^{\lambda} Q_{\lambda \alpha \beta} .
\end{aligned}
$$

\section{GENERALIZED OFF-SHELL ADT CURRENT AND POTENTIAL IN PALATINI THEORY}

In this section we construct the off-shell ADT current and potential in Palatini theory of gravity with torsion and non-metricity. In Palatini formalism, the two independent dynamic fields are the metric $g_{\mu \nu}$ and the affine connection $\Gamma_{\mu \nu}^{\lambda}$.

\section{A. Off-shell currents}

We consider a general Palatini theory of gravity with action

$$
I[g, \Gamma]=\frac{1}{16 \pi G} \int d^{D} x \sqrt{-g} L(g, \Gamma) .
$$

In this paper, we suppose that the theories under consideration are diffeomorhism invariant

up to a boundary term. For convenience, we denote the dynamic fields jointly as $\Phi=$ $\left(g_{\mu \nu}, \Gamma_{\mu \nu}^{\lambda}\right)$. The generic variation of the Lagrangian leads to

$$
\begin{aligned}
\delta(\sqrt{-g} L) & =\sqrt{-g} \mathcal{E}_{\Phi} \delta \Phi+\partial_{\mu}\left(\sqrt{-g} \Theta^{\mu}(\delta \Phi, \Phi)\right) \\
& =-\sqrt{-g} \mathcal{E}^{\mu \nu} \delta g_{\mu \nu}+\sqrt{-g} P_{\lambda}^{\nu \mu} \delta \Gamma_{\mu \nu}^{\lambda}+\partial_{\mu} \tilde{\Theta}^{\mu}(\delta \Phi, \Phi),
\end{aligned}
$$

where $\mathcal{E}_{\Phi}=\left(\mathcal{E}_{\mu \nu}, P_{\lambda}^{\nu \mu}\right)$ and $\tilde{\Theta}^{\mu}=\sqrt{-g} \Theta^{\mu}$ denote the Euler-Lagrange expression and the surface term, respectively. And hereinafter, the tilde ${ }^{\sim}$ over a letter denotes $\tilde{X}=\sqrt{-g} X$.

For an arbitrary smooth vector field $\xi=\xi^{\mu} \partial_{\mu}$ defined over the spacetime, which can generate the diffeomorhism $x^{\mu} \rightarrow x^{\mu}-\xi^{\mu}$, the diffeomorphism variation of the Lagrangian is given by

$$
\begin{aligned}
\delta_{\xi}(\sqrt{-g} L) & =\sqrt{-g} \mathcal{E}_{\Phi} \delta_{\xi} \Phi+\partial_{\mu} \tilde{\Theta}^{\mu}\left(\delta_{\xi} \Phi, \Phi\right) \\
& =-\sqrt{-g} \mathcal{E}^{\mu \nu} \delta_{\xi} g_{\mu \nu}+\sqrt{-g} P_{\lambda}{ }^{\nu \mu} \delta_{\xi} \Gamma_{\mu \nu}^{\lambda}+\partial_{\mu} \tilde{\Theta}^{\mu}\left(\delta_{\xi} \Phi, \Phi\right),
\end{aligned}
$$


where the diffeomorphism transformations have the forms [31]

$$
\begin{aligned}
\delta_{\xi} g_{\mu \nu} & =\xi^{\sigma} \partial_{\sigma} g_{\mu \nu}+g_{\sigma \nu} \partial_{\mu} \xi^{\sigma}+g_{\mu \sigma} \partial_{\nu} \xi^{\sigma} \\
& =2 \nabla_{(\mu} \xi_{\nu)}+2 N_{(\mu \nu)}^{\lambda} \xi_{\lambda} \\
\delta_{\xi} \Gamma_{\mu \nu}^{\lambda} & =\partial_{\mu} \partial_{\nu} \xi^{\lambda}+\xi^{\sigma} \partial_{\sigma} \Gamma_{\mu \nu}^{\lambda}-\Gamma_{\mu \nu}^{\sigma} \partial_{\sigma} \xi^{\lambda}+\Gamma_{\sigma \nu}^{\lambda} \partial_{\mu} \xi^{\sigma}+\Gamma_{\mu \sigma}^{\lambda} \partial_{\nu} \xi^{\sigma} \\
& =\nabla_{\mu} \bar{\nabla}_{\nu} \xi^{\lambda}-R_{\nu \mu \sigma}^{\lambda} \xi^{\sigma} .
\end{aligned}
$$

Then, one has

$$
\begin{aligned}
\delta_{\xi}(\sqrt{-g} L) & =\left[2 \nabla_{\mu}\left(\sqrt{-g} \mathcal{E}^{(\mu \nu)}\right)-2 T_{\mu}\left(\sqrt{-g} \mathcal{E}^{(\mu \nu)}\right)-2 \sqrt{-g} \mathcal{E}^{(\mu \lambda)} N^{\nu}{ }_{\mu \lambda}-\sqrt{-g} P_{\lambda}{ }^{\alpha \beta} R^{\lambda}{ }_{\alpha \beta}{ }^{\nu}\right] \xi_{\nu} \\
& +\left[\bar{\nabla}_{\nu}\left[\nabla_{\mu}\left(\sqrt{-g} P_{\sigma}{ }^{\nu \mu}\right)-T_{\mu}\left(\sqrt{-g} P_{\sigma}{ }^{\nu \mu}\right)\right]+T_{\nu}\left[\nabla_{\mu}\left(\sqrt{-g} P_{\sigma}{ }^{\nu \mu}\right)-T_{\mu}\left(\sqrt{-g} P_{\sigma}{ }^{\nu \mu}\right)\right]\right] \xi^{\sigma} \\
& +\partial_{\mu}\left(\tilde{\Theta}^{\mu}\left(\delta_{\xi} \Phi, \Phi\right)-2 \tilde{S}^{\mu}\left(\delta_{\xi} \Phi, \Phi\right)\right),
\end{aligned}
$$

where

$$
2 \tilde{S}^{\mu}\left(\delta_{\xi} \Phi, \Phi\right) \equiv 2 \sqrt{-g} \mathcal{E}^{(\mu \nu)} \xi_{\nu}-\sqrt{-g} P_{\sigma}{ }^{\nu \mu} \bar{\nabla}_{\nu} \xi^{\sigma}+\left[\nabla_{\nu}\left(\sqrt{-g} P_{\sigma}^{\mu \nu}\right)-T_{\nu}\left(\sqrt{-g} P_{\sigma}^{\mu \nu}\right)\right] \xi^{\sigma}
$$

On the other hand, the diffeomorphism variation of the Lagrangian has the form

$$
\delta_{\xi}(\sqrt{-g} L)=\partial_{\mu}\left(\xi^{\mu} \sqrt{-g} L\right)
$$

By equating the two expressions above, we can obtain an off-shell identity that can be thought of as generalized Bianchi identity in Palatini gravity

$$
\begin{aligned}
& {\left[2 \nabla_{\mu}\left(\sqrt{-g} \mathcal{E}^{(\mu \nu)}\right)-2 T_{\mu}\left(\sqrt{-g} \mathcal{E}^{(\mu \nu)}\right)-2 \sqrt{-g} \mathcal{E}^{(\mu \lambda)} N^{\nu}{ }_{\mu \lambda}-\sqrt{-g} P_{\lambda}{ }^{\alpha \beta} R^{\lambda}{ }_{\alpha \beta}{ }^{\nu}\right] \xi_{\nu}} \\
& +\left[\bar{\nabla}_{\nu}\left[\nabla_{\mu}\left(\sqrt{-g} P_{\sigma}^{\nu \mu}\right)-T_{\mu}\left(\sqrt{-g} P_{\sigma}^{\nu \mu}\right)\right]+T_{\nu}\left[\nabla_{\mu}\left(\sqrt{-g} P_{\sigma}^{\nu \mu}\right)-T_{\mu}\left(\sqrt{-g} P_{\sigma}^{\nu \mu}\right)\right]\right] \xi^{\sigma} \\
& =\partial_{\mu}\left(\sqrt{-g} \mathcal{Z}_{\xi}^{\mu}\right)
\end{aligned}
$$

where we have defined

$$
\sqrt{-g} \mathcal{Z}_{\xi}^{\mu} \equiv \xi^{\mu} \sqrt{-g} L-\tilde{\Theta}^{\mu}\left(\delta_{\xi} \Phi, \Phi\right)+2 \tilde{S}^{\mu}\left(\delta_{\xi} \Phi, \Phi\right)+\partial_{\nu} \tilde{U}^{[\mu \nu]}
$$

and $\tilde{U}^{\mu \nu}=\tilde{U}^{[\mu \nu]}$ is an arbitrary anti-symmetric second rank tensor that will be dropped out in what follows since this ambiguity will not affect the final conserved charges.

Furthermore, from Eqs.(15), (17) and (20) we obtain

$$
\partial_{\mu}\left(2 \sqrt{-g} \mathrm{E}_{\xi}^{\mu}\right)=\sqrt{-g} \mathcal{E}^{(\mu \nu)} \delta_{\xi} g_{\mu \nu}-\sqrt{-g} P_{\lambda}^{\nu \mu} \delta_{\xi} \Gamma_{\mu \nu}^{\lambda}=-\sqrt{-g} \mathcal{E}_{\Phi} \delta_{\xi} \Phi,
$$


where

$$
\mathrm{E}_{\xi}^{\mu} \equiv S_{\xi}^{\mu}-\frac{1}{2} \mathcal{Z}_{\xi}^{\mu}
$$

If the transformation $\delta_{\xi} \Phi$ is an exact symmetry $\delta_{\xi} \Phi=0$ [10 12] (i.e. $\delta_{\xi} g_{\mu \nu}=0$ and $\delta_{\xi} \Gamma_{\mu \nu}^{\lambda}=$ 0 , in which the vector field $\xi$ satisfying these two conditions is also called the generalized Killing vector [31]]), we can get

$$
\partial_{\mu}\left(\sqrt{-g} \mathrm{E}_{\xi}^{\mu}\right)=0
$$

To obtain the off-shell conserved current, we consider the double variations [23]

$$
\delta_{1} \delta_{2} I[\Phi]=\frac{1}{16 \pi G} \int d^{D} x\left[\delta_{1}\left(\sqrt{-g} \mathcal{E}_{\Phi} \delta_{2} \Phi\right)+\partial_{\mu}\left(\delta_{1} \tilde{\Theta}^{\mu}\left(\delta_{2} \Phi, \Phi\right)\right)\right]
$$

Using the property

$$
\left(\delta_{1} \delta_{2}-\delta_{2} \delta_{1}\right) I[\Phi]=0
$$

in which we take one of the variations as a diffeomorhism variation $\delta_{\xi}$ (In this case, the property (26) is trivially true, since the action should not vary under a diffeomorhism), we have

$$
0=\frac{1}{16 \pi G} \int d^{D} x\left[\delta_{\xi}\left(\sqrt{-g} \mathcal{E}_{\Phi} \delta \Phi\right)-\delta\left(\sqrt{-g} \mathcal{E}_{\Phi} \delta_{\xi} \Phi\right)+\partial_{\mu} \tilde{\omega}^{\mu}\left(\delta \Phi, \delta_{\xi} \Phi, \Phi\right)\right]
$$

where we have used the symplectic current definition in CPSM [7-9]

$$
\tilde{\omega}^{\mu}\left(\delta \Phi, \delta_{\xi} \Phi, \Phi\right)=\delta \tilde{\Theta}^{\mu}\left(\delta_{\xi} \Phi, \Phi\right)-\delta_{\xi} \tilde{\Theta}^{\mu}(\delta \Phi, \Phi)
$$

We assume that the spacetime is endowed with a generalized Killing vector i.e. $\delta_{\xi} \Phi=0$, then $\tilde{\omega}^{\mu}\left(\delta \Phi, \delta_{\xi} \Phi, \Phi\right)=0$. Since the Euler-Lagrange expression is covariant, we can obtain the off-shell identity ${ }^{1}$

$$
\delta_{\xi}\left(\sqrt{-g} \mathcal{E}_{\Phi} \delta \Phi\right)=\partial_{\mu}\left(\xi^{\mu} \sqrt{-g} \mathcal{E}_{\Phi} \delta \Phi\right)=0
$$

Similar to [23, 24], we can introduce an off-shell ADT current for the generalized Killing vector

$$
\sqrt{-g} \mathcal{J}_{\mathrm{ADT}}^{\mu}=\delta\left(\sqrt{-g} \mathrm{E}_{\xi}^{\mu}\right)+\frac{1}{2} \sqrt{-g} \xi^{\mu} \mathcal{E}_{\Phi} \delta \Phi,
$$

where we have taken $\delta \xi^{\mu}=0$, i.e. the generators are field-independent. From Eqs.(24) and (29), it is easy to see the conservation of the off-shell ADT current as

$$
\partial_{\mu}\left(\sqrt{-g} \mathcal{J}_{\mathrm{ADT}}^{\mu}\right)=0
$$

\footnotetext{
1 That is, the identity (29) holds not only on-shell but also off-shell.
} 
So we are allowed to introduce the off-shell ADT potential $\mathcal{Q}_{\mathrm{ADT}}^{\mu \nu}$ as

$$
\sqrt{-g} \mathcal{J}_{\mathrm{ADT}}^{\mu}=\partial_{\nu}\left(\sqrt{-g} \mathcal{Q}_{\mathrm{ADT}}^{\mu \nu}\right)
$$

\section{B. Off-shell potentials}

In this subsection we construct the off-shell ADT potential by using the off-shell Noether current and potential. From Eqs.(15) and (19), and using the off-shell identity (22), we can introduce the off-shell Noether current

$$
J_{\xi}^{\mu}=2 \sqrt{-g} \mathrm{E}_{\xi}^{\mu}+\sqrt{-g} \xi^{\mu} L-\tilde{\Theta}^{\mu}\left(\delta_{\xi} \Phi, \Phi\right)
$$

which satisfies $\partial_{\mu} J_{\xi}^{\mu}=0$. Then, the off-shell Noether potential $K_{\xi}^{\mu \nu}$ can be introduced as

$$
J_{\xi}^{\mu}=\partial_{\nu} K_{\xi}^{\mu \nu}
$$

where $J_{\xi}^{\mu}=\sqrt{-g} \mathcal{J}_{\xi}^{\mu}$ and $K_{\xi}^{\mu \nu}=\sqrt{-g} \mathcal{K}_{\xi}^{\mu \nu}$. The diffeomorphism variation of the surface term is

$$
\delta_{\xi} \tilde{\Theta}^{\mu}(\delta \Phi, \Phi)=\mathcal{L}_{\xi} \tilde{\Theta}^{\mu}(\delta \Phi, \Phi)=\xi^{\nu} \partial_{\nu} \tilde{\Theta}^{\mu}-\tilde{\Theta}^{\nu} \partial_{\nu} \xi^{\mu}+\tilde{\Theta}^{\mu} \partial_{\nu} \xi^{\nu}
$$

which leads to

$$
\xi^{\mu} \partial_{\nu} \tilde{\Theta}^{\nu}(\delta \Phi, \Phi)=\partial_{\nu}\left(2 \xi^{[\mu} \tilde{\Theta}^{\nu]}(\delta \Phi, \Phi)\right)+\mathcal{L}_{\xi} \tilde{\Theta}^{\mu}(\delta \Phi, \Phi)
$$

By varying the off-shell Noether current (33) and using the off-shell ADT current (30), we get

$$
\delta J_{\xi}^{\mu}=2 \sqrt{-g} \mathcal{J}_{\mathrm{ADT}}^{\mu}+\partial_{\nu}\left(2 \xi^{[\mu} \tilde{\Theta}^{\nu]}(\delta \Phi, \Phi)\right)-\tilde{\omega}^{\mu}\left(\delta \Phi, \delta_{\xi} \Phi, \Phi\right),
$$

where we have used Eq.(36) and the symplectic current definition (28). From Eq.(37) and the variation of (34) $\delta J_{\xi}^{\mu}=\partial_{\nu}\left(\delta K_{\xi}^{\mu \nu}\right)$ we obtain

$$
\begin{aligned}
2 \sqrt{-g} \mathcal{J}_{\mathrm{ADT}}^{\mu} & =\partial_{\nu}\left(\delta K_{\xi}^{\mu \nu}-2 \xi^{[\mu} \tilde{\Theta}^{\nu]}(\delta \Phi, \Phi)\right)+\tilde{\omega}^{\mu}\left(\delta \Phi, \delta_{\xi} \Phi, \Phi\right) \\
& =\partial_{\nu}\left(2 \sqrt{-g} \mathcal{Q}_{\mathrm{ADT}}^{\mu \nu}\right)
\end{aligned}
$$

where $\delta_{\xi} \Phi=0, \tilde{\omega}^{\mu}\left(\delta \Phi, \delta_{\xi} \Phi, \Phi\right)=0$, for exact symmetry. $\mathcal{Q}_{\mathrm{ADT}}^{\mu \nu}$ is the off-shell ADT potential corresponding to the off-shell ADT current $\mathcal{J}_{\mathrm{ADT}}^{\mu}$, which is given by

$$
2 \sqrt{-g} \mathcal{Q}_{\mathrm{ADT}}^{\mu \nu}=\delta K_{\xi}^{\mu \nu}-2 \xi^{[\mu} \tilde{\Theta}^{\nu]}(\delta \Phi, \Phi)
$$


In order to obtain finite conserved charges, we use the one parameter path integral method [9, 17-20] in the space of solutions when the gravity theory has black hole solutions. By assuming that the integral is path-independent, we can define the off-shell ADT conserved charge as

$$
\begin{aligned}
\mathcal{Q}(\xi) & \equiv \frac{1}{8 \pi G} \int_{0}^{1} d s \int_{\Sigma} d^{D-2} x_{\mu \nu} \sqrt{-g} \mathcal{Q}_{\mathrm{ADT}}^{\mu \nu} \\
& =\frac{1}{16 \pi G} \int_{\Sigma} d^{D-2} x_{\mu \nu}\left[\Delta K^{\mu \nu}(\xi)-2 \xi^{[\mu} \int_{0}^{1} d s \tilde{\Theta}^{\nu]}(\Phi, s \mathcal{M})\right],
\end{aligned}
$$

where

$$
\Delta K^{\mu \nu}=K_{s=1}^{\mu \nu}-K_{s=0}^{\mu \nu}
$$

is the finite difference of Noether potential between the given solution and the background solution, $s$ is the path parameter $(s \in[0,1])$, and $\mathcal{M}$ 's are the black hole solution parameters. Eq.(40) can be used to compute quasi-local conserved charges including mass, angular momentum and entropy for a given black hole solution.

\section{OFF-SHELL ADT POTENTIALS IN GENERAL MODELS}

\section{A. Einstein-Hilbert theory in Palatini formalism}

In this section we will give the off-shell ADT potential of Einstein-Hilbert theory in Palatini formalism with tortion and non-metricity. The Einstein-Hilbert action with a cosmological constant in D-dimension is given by

$$
I[g, \Gamma]=\frac{1}{16 \pi G} \int d^{D} x \sqrt{-g}(R-2 \Lambda)
$$

From Eq.(14), the variations of the Einstein-Hilbert action give the Euler-Lagrange expressions and the surface term as

$$
\begin{aligned}
\mathcal{E}^{(\mu \nu)} & =R^{(\mu \nu)}-\frac{1}{2} R g^{\mu \nu}+\Lambda g^{\mu \nu} \\
P_{\lambda}{ }^{\nu \mu} & =-\frac{\nabla_{\lambda}\left(\sqrt{-g} g^{\nu \mu}\right)}{\sqrt{-g}}+\frac{\nabla_{\sigma}\left(\sqrt{-g} g^{\nu \sigma}\right) \delta_{\lambda}^{\mu}}{\sqrt{-g}}+\left(T_{\lambda} g^{\nu \mu}-T^{\nu} \delta_{\lambda}^{\mu}+g^{\nu \sigma} T_{\sigma \lambda}^{\mu}\right) \\
\tilde{\Theta}^{\lambda} & =\sqrt{-g} g^{\mu \nu} \delta \Gamma_{\mu \nu}^{\lambda}-\sqrt{-g} g^{\nu \lambda} \delta_{\sigma}^{\mu} \delta \Gamma_{\mu \nu}^{\sigma} .
\end{aligned}
$$


The diffeomorphism variation of Lagrangian leads to

$$
\begin{aligned}
\tilde{\Theta}^{\lambda}\left(\delta_{\xi} \Phi, \Phi\right) & =\sqrt{-g} g^{\mu \nu} \delta_{\xi} \Gamma_{\mu \nu}^{\lambda}-\sqrt{-g} g^{\nu \lambda} \delta_{\sigma}^{\mu} \delta_{\xi} \Gamma_{\mu \nu}^{\sigma} \\
& =\partial_{\nu}\left(\sqrt{-g} P_{\sigma}{ }^{\nu \lambda} \xi^{\sigma}\right)-T_{\nu}\left(\sqrt{-g} P_{\sigma}{ }^{\nu \lambda} \xi^{\sigma}\right)+\sqrt{-g} \Gamma_{\rho \nu}^{\lambda} P_{\sigma}^{\nu \rho} \xi^{\sigma}-\bar{\nabla}_{\nu}\left(\sqrt{-g} P_{\sigma}{ }^{\nu \lambda}\right) \xi^{\sigma} .
\end{aligned}
$$

By using the Bianchi identities (9)-(10), we can prove that the left hand side of Eq.(20) vanishes in Einstein-Hilbert theory, which leads to $\mathcal{Z}_{\xi}^{\mu}=0$. From Eq.(33), we get the off-shell Noether current

$$
\begin{aligned}
J_{\xi}^{\mu}= & 2 \sqrt{-g} R^{(\mu \nu)} \xi_{\nu}+\sqrt{-g} \check{R}^{\mu \nu} \xi_{\nu}-\sqrt{-g} R^{\mu \nu} \xi_{\nu}+\left[\nabla_{\nu}\left(\sqrt{-g} P_{\sigma}^{\mu \nu}\right)-T_{\nu}\left(\sqrt{-g} P_{\sigma}^{\mu \nu}\right)\right] \xi^{\sigma} \\
& +\partial_{\nu}\left(2 \sqrt{-g} g^{\sigma[\mu} \bar{\nabla}_{\sigma} \xi^{\nu]}\right) .
\end{aligned}
$$

By using the Bianchi identities (91)-(10) again, the first line of Eq.(47) vanishes, then the off-shell Noether current and potential are

$$
\begin{gathered}
J_{\xi}^{\mu}=\partial_{\nu}\left(2 \sqrt{-g} g^{\sigma[\mu} \bar{\nabla}_{\sigma} \xi^{\nu]}\right) \\
=\partial_{\nu}\left(\sqrt{-g} \mathcal{K}_{\xi}^{\mu \nu}\right), \\
\mathcal{K}_{\xi}^{\mu \nu}=2 \bar{\nabla}^{[\mu} \xi^{\nu]} .
\end{gathered}
$$

From Eqs.(39) and (45) we obtain the final expression of the off-shell ADT potential

$$
\begin{aligned}
\mathcal{Q}_{\mathrm{ADT}}^{\mu \nu}= & \frac{1}{2} h \bar{\nabla}^{[\mu} \xi^{\nu]}-h^{\sigma[\mu} \bar{\nabla}_{\sigma} \xi^{\nu]}+g^{\sigma[\mu} \delta \Gamma_{\rho \sigma}^{\nu]} \xi^{\rho} \\
& -\xi^{[\mu} \delta \Gamma^{\nu]}{ }_{\alpha \beta} g^{\alpha \beta}+\xi^{[\mu} g^{\nu] \sigma} \delta \Gamma_{\rho \sigma}^{\rho},
\end{aligned}
$$

where we have defined

$$
h_{\mu \nu}=\delta g_{\mu \nu}, \quad h^{\mu \nu}=g^{\mu \alpha} g^{\nu \beta} \delta g_{\alpha \beta}=-\delta g^{\mu \nu}, \quad h=g^{\mu \nu} \delta g_{\mu \nu} .
$$

From Eq.(50), we see that the torsion and non-metricity tensors do not apparently present in the off-shell ADT potential, but included through the affine connection $\Gamma_{\mu \nu}^{\lambda}$. On the other hand, our formalism does not match with the BBC formalism in Palatini formalism of general relativity presented in [35] when the torsion are set to zero. As pointed out in [35] this difference in Palatini theory comes from the use of the generalized Killing vector.

In the standard metric formalism, it is assumed that torsion and non-metricity are absent, i.e. $\nabla_{\alpha} g_{\mu \nu}=0, T_{\mu \nu}^{\lambda}=-2 \Gamma_{[\mu \nu]}^{\lambda}=0, \bar{\nabla}_{\mu}=\nabla_{\mu}$, and the connection is completely fixed to 
Levi-Civita connection $\stackrel{\circ}{\Gamma}^{\lambda}{ }_{\mu \nu}$. Then, our expressions reduce to

$$
\begin{aligned}
& \mathcal{K}_{\xi}^{\mu \nu}=2 \nabla^{[\mu} \xi^{\nu]}, \\
& \Theta^{\mu}(\delta \Phi, \Phi)=2 \nabla^{[\sigma} h^{\mu]}, \\
& \mathcal{Q}_{\mathrm{ADT}}^{\mu \nu}=\frac{1}{2} h \nabla^{[\mu} \xi^{\nu]}-h^{\sigma[\mu} \nabla_{\sigma} \xi^{\nu]}-\xi^{[\mu} \nabla_{\sigma} h^{\nu] \sigma}+\xi_{\sigma} \nabla^{[\mu} h^{\nu] \sigma}+\xi^{[\mu} \nabla^{\nu]} h,
\end{aligned}
$$

which are well known results in Einstein general relativity in the metric formalism [7 9$]$.

\section{B. General $L\left(g_{\mu \nu}, R_{\nu \alpha \mu}^{\lambda}, T_{\alpha \beta}^{\lambda}, Q_{\alpha \mu \nu}\right)$ theories}

In the gravitation sector, the most general Lagrangian that one could write down (without including additional tensors constructed by the covariant derivatives of basic geometrical objects) is $L\left(g_{\mu \nu}, R^{\lambda}{ }_{\nu \alpha \mu}, T^{\lambda}{ }_{\alpha \beta}, Q_{\alpha \mu \nu}\right)$ [36]. In this subsection we will derive the off-shell ADT potential in this theory. The action is given by

$$
I[g, \Gamma]=\frac{1}{16 \pi G} \int d^{D} x \sqrt{-g} L\left(g_{\mu \nu}, R_{\nu \alpha \mu}^{\lambda}, T_{\alpha \beta}^{\lambda}, Q_{\alpha \mu \nu}\right) .
$$

The EOMs have been given in [36] for this theory. In order to derive the off-shell ADT potential we write them down and give the surface term by generic variation

$$
\begin{aligned}
\mathcal{E}^{\mu \nu} & =-\mathcal{G}^{\mu \nu}-\frac{1}{2} g^{\mu \nu} L+T_{\sigma} W^{\sigma \mu \nu}-\frac{1}{\sqrt{-g}} \nabla_{\sigma}\left(\sqrt{-g} W^{\sigma \mu \nu}\right), \\
P_{\lambda}{ }^{\nu \mu} & =-\frac{2}{\sqrt{-g}} \nabla_{\sigma}\left(\sqrt{-g} \Omega_{\lambda}{ }^{\nu \sigma \mu}\right)-\Omega_{\lambda}{ }^{\nu \sigma \rho} T_{\sigma \rho}^{\mu}-2 V_{\lambda}{ }^{\mu \nu}+2 W_{\lambda}^{\mu \nu}+2 T_{\sigma} \Omega_{\lambda}{ }^{\nu \sigma \mu}, \\
\tilde{\Theta}^{\alpha}(\delta \Phi, \Phi) & =2 \sqrt{-g} \Omega_{\lambda}{ }^{\nu \alpha \mu} \delta \Gamma^{\lambda}{ }_{\mu \nu}-\sqrt{-g} W^{\alpha \mu \nu} \delta g_{\mu \nu},
\end{aligned}
$$

where

$$
\begin{aligned}
\mathcal{G}^{\mu \nu} & \equiv \frac{\partial L}{\partial g_{\mu \nu}}, & W^{\alpha \mu \nu} & =W^{\alpha(\mu \nu)} \equiv \frac{\partial L}{\partial Q_{\alpha \mu \nu}} \\
\Omega_{\lambda}{ }^{\nu \alpha \mu} & =\Omega_{\lambda}{ }^{\nu[\alpha \mu]} \equiv \frac{\partial L}{\partial R^{\lambda}{ }_{\nu \alpha \mu}}, & V_{\lambda}^{\mu \nu} & =V_{\lambda}{ }^{[\mu \nu]} \equiv \frac{\partial L}{\partial T^{\lambda}{ }_{\mu \nu}} .
\end{aligned}
$$

The diffeomorphism variation of the Lagrangian has the form

$$
\begin{aligned}
\delta_{\xi}(\sqrt{-g} L) & =\left[2 \nabla_{\mu}\left(\sqrt{-g} \mathcal{E}^{(\mu \nu)}\right)-2 T_{\mu}\left(\sqrt{-g} \mathcal{E}^{(\mu \nu)}\right)-2 \sqrt{-g} \mathcal{E}^{(\mu \lambda)} N^{\nu}{ }_{\mu \lambda}-\sqrt{-g} P_{\lambda}{ }^{\alpha \beta} R^{\lambda}{ }_{\alpha \beta}{ }^{\nu}\right] \xi_{\nu} \\
& +\left[\bar{\nabla}_{\nu}\left[\nabla_{\mu}\left(\sqrt{-g} P_{\sigma}{ }^{\nu \mu}\right)-T_{\mu}\left(\sqrt{-g} P_{\sigma}{ }^{\nu \mu}\right)\right]+T_{\nu}\left[\nabla_{\mu}\left(\sqrt{-g} P_{\sigma}{ }^{\nu \mu}\right)-T_{\mu}\left(\sqrt{-g} P_{\sigma}{ }^{\nu \mu}\right)\right]\right] \xi^{\sigma} \\
& +\partial_{\mu}\left(\tilde{\Theta}^{\mu}\left(\delta_{\xi} \Phi, \Phi\right)-2 \tilde{S}^{\mu}\left(\delta_{\xi} \Phi, \Phi\right)\right) .
\end{aligned}
$$


For a general Palatini theory of gravity, which is diffeomorphism invariant up to a boundary term, the first two lines must vanish

$$
\begin{aligned}
& {\left[2 \nabla_{\mu}\left(\sqrt{-g} \mathcal{E}^{(\mu \nu)}\right)-2 T_{\mu}\left(\sqrt{-g} \mathcal{E}^{(\mu \nu)}\right)-2 \sqrt{-g} \mathcal{E}^{(\mu \lambda)} N_{\mu \lambda}^{\nu}-\sqrt{-g} P_{\lambda}{ }^{\alpha \beta} R^{\lambda}{ }_{\alpha \beta}{ }^{\nu}\right] \xi_{\nu}} \\
& +\left[\bar{\nabla}_{\nu}\left[\nabla_{\mu}\left(\sqrt{-g} P_{\sigma}{ }^{\nu \mu}\right)-T_{\mu}\left(\sqrt{-g} P_{\sigma}{ }^{\nu \mu}\right)\right]+T_{\nu}\left[\nabla_{\mu}\left(\sqrt{-g} P_{\sigma}{ }^{\nu \mu}\right)-T_{\mu}\left(\sqrt{-g} P_{\sigma}{ }^{\nu \mu}\right)\right]\right] \xi^{\sigma} \\
& =0
\end{aligned}
$$

which can be thought of as the generalized Bianchi identity in $L\left(g_{\mu \nu}, R_{\nu \alpha \mu}^{\lambda}, T_{\alpha \beta}^{\lambda}, Q_{\alpha \mu \nu}\right)$ theory. From Eqs.(33) and (34) we can get the off-shell Noether current and potential as

$$
\begin{aligned}
J_{\xi}^{\mu} & =\partial_{\nu}\left[2 \sqrt{-g}\left(V_{\lambda}{ }^{\mu \nu} \xi^{\lambda}-\Omega_{\lambda}{ }^{\sigma \mu \nu} \bar{\nabla}_{\sigma} \xi^{\lambda}\right)\right] \\
& =\partial_{\nu}\left[\sqrt{-g} \mathcal{K}_{\xi}^{\mu \nu}\right] \\
\mathcal{K}_{\xi}^{\mu \nu} & =2\left(V_{\lambda}{ }^{\mu \nu} \xi^{\lambda}-\Omega_{\lambda}{ }^{\sigma \mu \nu} \bar{\nabla}_{\sigma} \xi^{\lambda}\right) .
\end{aligned}
$$

From Eq.(39), we can get the off-shell ADT potential

$$
\begin{aligned}
\mathcal{Q}_{\mathrm{ADT}}^{\mu \nu} & =\frac{1}{2} h{V_{\lambda}}^{\mu \nu} \xi^{\lambda}+\left(\delta V_{\lambda}{ }^{\mu \nu}\right) \xi^{\lambda}-\Omega_{\lambda}{ }^{\sigma \mu \nu}\left(\frac{1}{2} h \bar{\nabla}_{\sigma} \xi^{\lambda}+\delta \Gamma_{\rho \sigma}^{\lambda} \xi^{\rho}\right) \\
& -\left(\delta \Omega_{\lambda}{ }^{\sigma \mu \nu}\right) \bar{\nabla}_{\sigma} \xi^{\lambda}-\xi^{[\mu}\left(2 \Omega_{\lambda}{ }^{|\beta| \nu] \alpha} \delta \Gamma_{\alpha \beta}^{\lambda}-W^{\nu] \alpha \beta} \delta g_{\alpha \beta}\right) .
\end{aligned}
$$

Taking $L=R$, we compute

$$
\Omega_{\lambda}{ }^{\mu \alpha \nu}=\frac{\partial R}{\partial R_{\mu \alpha \nu}^{\lambda}}=\delta^{\beta}{ }_{\gamma} g^{\kappa \rho} \frac{\partial R^{\gamma}{ }_{\kappa \beta \rho}}{\partial R^{\lambda}{ }_{\mu \alpha \nu}}=g^{\mu[\nu} \delta_{\lambda}^{\alpha]},
$$

where we have used the fact

$$
\frac{\partial R^{\gamma}{ }_{\kappa \beta \rho}}{\partial R^{\lambda}{ }_{\mu \alpha \nu}}=\delta_{\lambda}^{\gamma} \delta_{\kappa}^{\mu} \delta_{\beta}^{[\alpha} \delta_{\rho}^{\nu]}
$$

And by using the fact

$$
\frac{\partial R}{\partial g^{\mu \nu}}=R_{(\mu \nu)}
$$

the results reduce to the Einstein-Hilbert theory present in Eqs.(43)-(46) and Eqs.(48)-(150).

\section{Teleparallel Palatini theory}

As a typical example, in this section we consider the teleparallel Palatini theory. The Lagrangian has the form [37]

$$
\mathcal{L}_{\|}=\sqrt{-g} L_{\|}=\frac{1}{2} \sqrt{-g} \mathbb{T}+\lambda_{\alpha}^{\beta \mu \nu} R_{\beta \mu \nu}^{\alpha}+\lambda^{\alpha \mu \nu} Q_{\alpha \mu \nu}
$$


where $\lambda_{\alpha}^{\beta \mu \nu}$ and $\lambda^{\alpha \mu \nu}$ are the Lagrange multipliers, which constrain the teleparallelity and metricity for Teleparallel Equivalent of General Relativity (TEGR). The torsion scalar is defined as

$$
\mathbb{T}=S_{\alpha}{ }^{\mu \nu} T^{\alpha}{ }_{\mu \nu}
$$

where

$$
S_{\alpha}^{\mu \nu}=a T_{\alpha}^{\mu \nu}+b T_{\alpha}^{[\mu \nu}+c \delta_{\alpha}^{[\mu} T^{\nu]}
$$

is the superpotential tensor, in which $a, b$ and $c$ are arbitrary constants. Varying the Lagrangian with respect to the dynamic fields and the Lagrange multipliers, we get

$$
\begin{aligned}
\delta\left(\sqrt{-g} L_{\|}\right)= & \sqrt{-g} \mathcal{E}_{\Phi} \delta \Phi+\partial_{\mu} \tilde{\Theta}^{\mu}(\delta \Phi, \Phi, \lambda) \\
= & -\sqrt{-g} \mathcal{E}^{\mu \nu} \delta g_{\mu \nu}+\sqrt{-g} P_{\lambda}{ }^{\nu \mu} \delta \Gamma_{\mu \nu}^{\lambda} \\
& +R^{\alpha}{ }_{\beta \mu \nu} \delta \lambda_{\alpha}{ }^{\beta \mu \nu}+Q_{\alpha \mu \nu} \delta \lambda^{\alpha \mu \nu}+\partial_{\mu} \tilde{\Theta}^{\mu}(\delta \Phi, \Phi, \lambda) .
\end{aligned}
$$

In TEGR, the teleparallelity and the dynamics are constrained by Lagrange multipliers EOMs

$$
R_{\beta \mu \nu}^{\alpha}=0, \quad Q_{\alpha \mu \nu}=0
$$

If we employ the constraint condition (68) and do not require the EOMs of the dynamic fields $\mathcal{E}_{\Phi}=0$ to be satisfied (i.e. off-shell), the Noether potential and the ADT potential can be extracted by using Eqs.(馬) and (60)

$$
\begin{aligned}
& \mathcal{K}_{\xi}^{\mu \nu}=2\left(S_{\alpha}{ }^{\mu \nu} \xi^{\alpha}-\lambda_{\alpha}{ }^{\sigma \mu \nu} \bar{\nabla}_{\sigma} \xi^{\alpha}\right), \\
& \mathcal{Q}_{\mathrm{ADT}}^{\mu \nu}=\frac{1}{2} h S_{\alpha}{ }^{\mu \nu} \xi^{\alpha}+\left(\delta S_{\alpha}{ }^{\mu \nu}\right) \xi^{\alpha}-\lambda_{\alpha}{ }^{\sigma \mu \nu}\left(\frac{1}{2} h \bar{\nabla}_{\sigma} \xi^{\alpha}+\delta \Gamma_{\rho \sigma}^{\alpha} \xi^{\rho}\right) \\
& -\left(\delta \lambda_{\alpha}{ }^{\sigma \mu \nu}\right) \bar{\nabla}_{\sigma} \xi^{\alpha}-\xi^{[\mu}\left(2 \lambda_{\rho}^{|\beta| \nu] \alpha} \delta \Gamma_{\alpha \beta}^{\rho}-\lambda^{\nu] \alpha \beta} \delta g_{\alpha \beta}\right),
\end{aligned}
$$

where we have used the equalities

$$
V_{\alpha}{ }^{\mu \nu}=\frac{\partial L_{\|}}{\partial T^{\alpha}{ }_{\mu \nu}}=S_{\alpha}{ }^{\mu \nu}, \quad \Omega_{\alpha}{ }^{\beta \mu \nu}=\frac{\partial L_{\|}}{\partial R^{\alpha}{ }_{\beta \mu \nu}}=\lambda_{\alpha}{ }^{\beta \mu \nu}, \quad W^{\alpha \mu \nu}=\frac{\partial L_{\|}}{\partial Q_{\alpha \mu \nu}}=\lambda^{\alpha \mu \nu} .
$$

Since the Lagrange multipliers do not affect the dynamical field equation [37, 38], and thus will not affect the spacetime configuration and the black hole solutions, and will not affect the final conserved charges when the explicit black hole solutions are concerned, the Lagrange multipliers terms can be dropped out from the Noether and the ADT potentials, through 
which Eqs.(69) and (170) reduce to

$$
\begin{aligned}
& \mathcal{K}_{\xi}^{\mu \nu}=2 S_{\alpha}{ }^{\mu \nu} \xi^{\alpha}, \\
& \mathcal{Q}_{\mathrm{ADT}}^{\mu \nu}=\frac{1}{2} h S_{\alpha}{ }^{\mu \nu} \xi^{\alpha}+\left(\delta S_{\alpha}{ }^{\mu \nu}\right) \xi^{\alpha} \text {. }
\end{aligned}
$$

These results are exactly those obtained in [33] by the direct use of the Noether's theorem and in [34] by Wald's CPSM in TEGR.

\section{CONSERVED CHARGES FOR EXPLICIT BLACK HOLE SOLUTIONS}

As thermodynamic systems, black holes have a characteristic temperature, an entropy and the laws of black hole thermodynamics associated with them. In Palatini theory of gravity with torsion and non-metricity, if the black hole solutions exist, we give the general expressions of the black hole conserved charges and the first law of black hole thermodynamics.

For simplicity, we consider a stationary axial-symmetric black hole solution with torsion and non-metricity. We adopt the coordinate in which the timelike and the rotational Killing vector are $\xi_{T}=\partial_{t}$ and $\xi_{R}=-\partial_{\varphi}$, respectively. The horizon Killing vector is a linear combination $\xi_{\mathrm{H}}=\partial_{t}+\Omega_{\mathrm{H}} \partial_{\varphi}$ that satisfies $\delta_{\xi} g_{\mu \nu}=0$ and $\delta_{\xi} \Gamma_{\mu \nu}^{\lambda}=0$, where $\Omega_{\mathrm{H}}$ is the horizon angular velocity. Similar to the CPSM and the BBC formalisms, we define the black hole entropy as the conserved charge associated with horizon Killing vector $\xi_{\mathrm{H}}$,

$$
\frac{\kappa}{2 \pi} \delta S_{\mathrm{H}}=\delta \mathcal{Q}\left(\xi_{\mathrm{H}}\right)=\frac{1}{8 \pi G} \int_{\mathcal{H}} d^{D-2} x_{\mu \nu} \sqrt{-g} \mathcal{Q}_{\mathrm{ADT}}^{\mu \nu}\left(\xi_{\mathrm{H}}\right),
$$

where $\kappa$ is the surface gravity defined as $\kappa=-n^{\nu} \xi^{\mu} \nabla_{\nu} \xi_{\mu}$ in the presence of torsion [39], leading to $T_{\mathrm{H}}=\kappa_{\mathrm{H}} / 2 \pi$. From the linearity of $\delta \mathcal{Q}(\xi)$ in $\xi$, we can obtain the first law

$$
T_{\mathrm{H}} \delta S_{\mathrm{H}}=\delta M-\Omega_{\mathrm{H}} \delta J
$$

where

$$
\delta M=\delta \mathcal{Q}\left(\xi_{T}\right)=\frac{1}{8 \pi G} \int_{\Sigma} d^{D-2} x_{\mu \nu} \sqrt{-g} \mathcal{Q}_{\mathrm{ADT}}^{\mu \nu}\left(\xi_{T}\right)
$$

and

$$
\delta J=\delta \mathcal{Q}\left(\xi_{R}\right)=\frac{1}{8 \pi G} \int_{\Sigma} d^{D-2} x_{\mu \nu} \sqrt{-g} \mathcal{Q}_{\mathrm{ADT}}^{\mu \nu}\left(\xi_{R}\right)
$$

are the mass and angular momentum variations associated with $\xi_{T}$ and $\xi_{R}$, respectively. Similar to SPSM [10 12] and our previous work [24], $\Sigma$ can be an almostly arbitrary smooth 
co-dimensional two surface surrounding the singularity. Our above formulation can be used to compute the conserved charges and derive the first law of black hole thermodynamics in the modified gravity theories with torsion and non-metricity (e.g. Palatini theory, MAG and TEGR etc.).

As a simple example, we now check the total mass of Schwarzschild black hole in the teleparallel theory of gravity. In spherical coordinates the Schwarzschild metric is given by

$$
d s^{2}=-\left(1-\frac{2 m}{r}\right) d t^{2}+\left(1-\frac{2 m}{r}\right)^{-1} d r^{2}+r^{2}\left(d \theta^{2}+\sin ^{2} \theta d \varphi^{2}\right) .
$$

It has the non-zero components of affine connection [33]

$$
\begin{array}{rlrl}
\Gamma_{t r}^{t} & =-\Gamma_{r r}^{r}=-\frac{m}{r^{2}}\left(1-\frac{2 m}{r}\right)^{-1}, & \Gamma_{\theta \theta}^{r}=r\left(1-\frac{2 m}{r}\right)^{1 / 2}, \\
\Gamma_{\varphi \varphi}^{r}=r\left(1-\frac{2 m}{r}\right)^{1 / 2} r \sin ^{2} \theta, & \Gamma_{r \theta}^{\theta}=\Gamma_{r \varphi}^{\varphi}=-\frac{1}{r}\left(1-\frac{2 m}{r}\right)^{-1 / 2}, \\
\Gamma_{\theta r}^{\theta}=\Gamma_{\varphi r}^{\varphi}=-\frac{1}{r}, & \Gamma_{\varphi \theta}^{\theta}=\sin \theta \cos \theta, \quad \Gamma_{\theta \varphi}^{\varphi}=\Gamma_{\varphi \theta}^{\varphi}=-\cot \theta,
\end{array}
$$

From the first formula of (3), we get the non-zero independent components of torsion tensor

$$
T_{t r}^{t}=\frac{m}{r^{2}}\left(1-\frac{2 m}{r}\right)^{-1}, \quad T_{r \theta}^{\theta}=T_{r \varphi}^{\varphi}=-\frac{1}{r}\left(1-\left(1-\frac{2 m}{r}\right)^{-1 / 2}\right) .
$$

From Eq.(66), the non-zero independent components of the superpotential are given by

$$
S_{t}^{t r}=\frac{1}{r}\left(1-\left(1-\frac{2 m}{r}\right)^{1 / 2}\right)-\frac{2 m}{r^{2}}, \quad S_{\theta}^{r \theta}=S_{\varphi}^{r \varphi}=-\frac{1}{2 r}\left(1-\left(1-\frac{2 m}{r}\right)^{1 / 2}\right)+\frac{m}{2 r^{2}} .
$$

By using Eqs.(40), (172), choosing the one parameter path by substituting $m$ by $s m$ in the Schwarzschild metric (78), choosing the timelike Killing vector $\xi^{\mu}=(-1,0,0,0)$, taking the integral surface $\Sigma: t=$ const $=r$ for convenience, and taking the limit $r \rightarrow \infty$, we get the total mass of Schwarzschild black hole as

$$
\begin{aligned}
M & =\frac{1}{8 \pi G} \lim _{r \rightarrow \infty} \int_{0}^{1} d s \int_{\Sigma} d^{D-2} x_{\mu \nu} \sqrt{-g} \mathcal{Q}_{\mathrm{ADT}}^{\mu \nu} \\
& =\frac{1}{16 \pi G} \lim _{r \rightarrow \infty} \int_{\Sigma} d^{2} x_{t r} 2 \sqrt{-g} S_{t}^{t r} \xi^{t} \\
& =\frac{m}{G} .
\end{aligned}
$$

Therefore, we get the correct conserved charge (mass) of Schwarzschild black hole in teleparallel theory of gravity by our generalized off-shell ADT conserved charge formalism. 


\section{CONCLUSIONS}

In this paper we have generalized the off-shell ADT conserved charge formalism to Palatini theory of gravity with torsion and non-metricity. For a general Palatini theory of gravity, which is diffeomorphism invariant up to a boundary term, we obtained the most general expression of off-shell ADT potential. As explicit examples, we derived the off-shell ADT potentials for Einstein-Hilbert action, the most general $L\left(g_{\mu \nu}, R_{\nu \alpha \mu}^{\lambda}, T_{\alpha \beta}^{\lambda}, Q_{\alpha \mu \nu}\right)$ theories and the teleparallel Palatini gravity.

In the non-Riemannian geometrical framework, if the black hole solutions exist for a gravity theory, we defined the black hole entropy as a conserved charge, and the first law of black hole thermodynamics was derived. It is easy to generalize our off-shell formalism to arbitrary diffeomorphism invariant theory of gravity with torsion and non-metricity, even including the matter field (i.e. the MAG) by using the 'exact symmetries'.

[1] R. L. Arnowitt, S. Deser and C. W. Misner, "The Dynamics of general relativity," Gen. Rel. Grav. 40 (2008) 1997 gr-qc/0405109].

[2] L. F. Abbott and S. Deser, "Stability of Gravity with a Cosmological Constant," Nucl. Phys. B 195 (1982) 76.

[3] L. F. Abbott and S. Deser, "Charge Definition in Nonabelian Gauge Theories," Phys. Lett. 116B (1982) 259.

[4] S. Deser and B. Tekin, "Gravitational energy in quadratic curvature gravities," Phys. Rev. Lett. 89 (2002) 101101 hep-th/0205318.

[5] S. Deser and B. Tekin, "Energy in generic higher curvature gravity theories," Phys. Rev. D 67 (2003) 084009 hep-th/0212292.

[6] C. Senturk, T. C. Sisman and B. Tekin, "Energy and Angular Momentum in Generic F(Riemann) Theories," Phys. Rev. D 86 (2012) 124030 arXiv:1209.2056 [hep-th]].

[7] R. M. Wald, "Black hole entropy is the Noether charge," Phys. Rev. D 48 (1993) no.8, R3427 gr-qc/9307038.

[8] V. Iyer and R. M. Wald, "Some properties of Noether charge and a proposal for dynamical black hole entropy," Phys. Rev. D 50 (1994) 846 gr-qc/9403028. 
[9] R. M. Wald and A. Zoupas, "A General definition of 'conserved quantities' in general relativity and other theories of gravity," Phys. Rev. D 61 (2000) 084027 gr-qc/9911095.

[10] K. Hajian and M. M. Sheikh-Jabbari, "Solution Phase Space and Conserved Charges: A General Formulation for Charges Associated with Exact Symmetries," Phys. Rev. D 93 (2016) no.4, 044074 arXiv:1512.05584 [hep-th]].

[11] K. Hajian, "Conserved charges and first law of thermodynamics for Kerr-de Sitter black holes," Gen. Rel. Grav. 48 (2016) no.8, 114 [arXiv:1602.05575 [gr-qc]].

[12] M. Ghodrati, K. Hajian and M. R. Setare, "Revisiting Conserved Charges in Higher Curvature Gravitational Theories,” Eur. Phys. J. C 76 (2016) no.12, 701 arXiv:1606.04353 [hep-th]].

[13] C. M. Chen, J. M. Nester and R. S. Tung, "Quasilocal energy momentum for gravity theories," Phys. Lett. A 203 (1995), 5-11 arXiv:gr-qc/9411048 [gr-qc]].

[14] C. M. Chen and J. M. Nester, "Quasilocal quantities for GR and other gravity theories," Class. Quant. Grav. 16 (1999), 1279-1304 arXiv:gr-qc/9809020 [gr-qc]].

[15] C. M. Chen, J. M. Nester and R. S. Tung, "The Hamiltonian boundary term and quasi-local energy flux," Phys. Rev. D 72 (2005), 104020 arXiv:gr-qc/0508026 [gr-qc]].

[16] C. M. Chen, J. M. Nester and R. S. Tung, "Gravitational energy for GR and Poincaré gauge theories: A covariant Hamiltonian approach,” Int. J. Mod. Phys. D 24 (2015) no.11, 1530026 arXiv:1507.07300 [gr-qc]].

[17] G. Barnich and F. Brandt, "Covariant theory of asymptotic symmetries, conservation laws and central charges," Nucl. Phys. B 633 (2002) 3 hep-th/0111246].

[18] G. Barnich, "Boundary charges in gauge theories: Using Stokes theorem in the bulk," Class. Quant. Grav. 20 (2003) 3685 hep-th/0301039].

[19] G. Barnich and G. Compere, "Generalized Smarr relation for Kerr AdS black holes from improved surface integrals," Phys. Rev. D 71 (2005) 044016 Erratum: [Phys. Rev. D 73 (2006) 029904] gr-qc/0412029].

[20] G. Barnich and G. Compere, "Surface charge algebra in gauge theories and thermodynamic integrability," J. Math. Phys. 49 (2008) 042901 [arXiv:0708.2378 [gr-qc]].

[21] A. Bouchareb and G. Clement, "Black hole mass and angular momentum in topologically massive gravity," Class. Quant. Grav. 24 (2007), 5581-5594 [arXiv:0706.0263 [gr-qc]].

[22] W. Kim, S. Kulkarni and S. H. Yi, "Quasilocal Conserved Charges in a Covariant Theory of Gravity," Phys. Rev. Lett. 111 (2013) no.8, 081101 Erratum: [Phys. Rev. Lett. 112 (2014) 
no.7, 079902] arXiv:1306.2138 [hep-th]].

[23] S. Hyun, J. Jeong, S. A. Park and S. H. Yi, "Quasilocal conserved charges and holography," Phys. Rev. D 90 (2014) no.10, 104016 [arXiv:1406.7101 [hep-th]].

[24] H. F. Ding and X. H. Zhai, "Generalized off-shell ADT conserved charges in the presence of matter Chern-Simons term," Class. Quant. Grav. 37 (2020) no.8, 085021 arXiv:1910.03864 [hep-th]].

[25] F. W. Hehl, J. D. McCrea, E. W. Mielke and Y. Ne'eman, "Metric affine gauge theory of gravity: Field equations, Noether identities, world spinors, and breaking of dilation invariance," Phys. Rept. 258 (1995) 1 gr-qc/9402012].

[26] B. Julia and S. Silva, "Currents and superpotentials in classical gauge invariant theories. 1. Local results with applications to perfect fluids and general relativity," Class. Quant. Grav. 15 (1998) 2173 gr-qc/9804029.

[27] B. Julia and S. Silva, "Currents and superpotentials in classical gauge theories: 2. Global aspects and the example of affine gravity," Class. Quant. Grav. 17 (2000) 4733 [gr-qc/0005127].

[28] G. Giachetta and G. Sardanashvily, "Stress energy momentum of affine metric gravity. Generalized Komar superpotential," Class. Quant. Grav. 13 (1996) L67 gr-qc/9511008.

G. Sardanashvily, "Stress - energy - momentum conservation law in gauge gravitation theory," Class. Quant. Grav. 14 (1997) 1357.

G. Giachetta, L. Mangiarotti and G. Sardanashvily, "Energy momentum and gauge conservation laws," Grav. Cosmol. 5 (1999) 92 gr-qc/9807054.

R. Hecht, F. W. Hehl, J. D. McCrea, E. W. Mielke and Y. Ne'eman, "Improved energy momentum currents in metric affine space-time," Phys. Lett. A 172 (1992) 13 [gr-qc/9210009]. R. Aros, M. Contreras, R. Olea, R. Troncoso and J. Zanelli, "Conserved charges for gravity with locally AdS asymptotics," Phys. Rev. Lett. 84 (2000) 1647 gr-qc/9909015].

R. Aros, M. Contreras, R. Olea, R. Troncoso and J. Zanelli, "Conserved charges for even dimensional asymptotically AdS gravity theories," Phys. Rev. D 62 (2000) 044002 hep-th/9912045.

H. Cebeci, O. Sarioglu and B. Tekin, "Negative mass solitons in gravity," Phys. Rev. D 73 (2006) 064020 hep-th/0602117.

Y. N. Obukhov and G. F. Rubilar, "Invariant conserved currents in gravity theories with local Lorentz and diffeomorphism symmetry," Phys. Rev. D 74 (2006) 064002 gr-qc/0608064. 
Y. N. Obukhov and G. F. Rubilar, "Invariant conserved currents in gravity theories: diffeomorphisms and local gauge symmetries," Phys. Rev. D $76 \quad$ (2007) 124030 arXiv:0712.3547 [hep-th]].

R. R. Lompay and A. N. Petrov, "Covariant Differential Identities and Conservation Laws in Metric-Torsion Theories of Gravitation. I. General Consideration," J. Math. Phys. 54 (2013) 062504 arXiv:1306.6887 [gr-qc]].

R. R. Lompay and A. N. Petrov, "Covariant Differential Identities and Conservation Laws in Metric-Torsion Theories of Gravitation. II. Manifestly Generally Covariant Theories," J. Math. Phys. 54 (2013) 102504 arXiv:1309.5620 [gr-qc]].

[29] D. N. Vollick, "Noether Charge and Black Hole Entropy in Modified Theories of Gravity," Phys. Rev. D 76 (2007) 124001 [arXiv:0710.1859 [gr-qc]].

[30] H. Adami, M. R. Setare, T. C. Sisman and B. Tekin, "Conserved Charges in Extended Theories of Gravity," Phys. Rept. 834 (2019) 1 [arXiv:1710.07252 [hep-th]].

[31] Y. N. Obukhov, F. Portales-Oliva, D. Puetzfeld and G. F. Rubilar, "Invariant conserved currents in generalized gravity," Phys. Rev. D 92 (2015) no.10, 104010 arXiv:1507.02191 [gr-qc]].

[32] S. Chakraborty and R. Dey, "Noether Current, Black Hole Entropy and Spacetime Torsion," Phys. Lett. B 786 (2018) 432 [arXiv:1806.05840 [gr-qc]].

[33] E. D. Emtsova, A. N. Petrov and A. V. Toporensky, "Conserved currents and superpotentials in teleparallel equivalent of GR," Class. Quant. Grav. 37 (2020) no.9, 095006 arXiv:1910.08960 [gr-qc]].

E. D. Emtsova, A. N. Petrov and A. V. Toporensky, "On conservation laws in teleparallel gravity," J. Phys. Conf. Ser. 1557 (2020) no.1, 012017.

[34] F. Hammad, D. Dijamco, A. Torres-Rivas and D. Bérubé, "Noether charge and black hole entropy in teleparallel gravity," Phys. Rev. D 100 (2019) no.12, 124040 arXiv:1912.08811 [gr-qc]].

[35] G. Barnich, P. Mao and R. Ruzziconi, "Conserved currents in the Palatini formulation of general relativity," PoS CORFU2019 (2020), 171 [arXiv:2004.15002 [gr-qc]].

[36] D. Iosifidis, "Metric-Affine Gravity and Cosmology/Aspects of Torsion and non-Metricity in Gravity Theories," arXiv:1902.09643 [gr-qc].

[37] J. Beltrán Jiménez, L. Heisenberg and T. S. Koivisto, "Teleparallel Palatini theories," JCAP 
08 (2018), 039 arXiv:1803.10185 [gr-qc]].

[38] A. Golovnev, T. Koivisto and M. Sandstad, "On the covariance of teleparallel gravity theories," Class. Quant. Grav. 34 (2017) no.14, 145013 [arXiv:1701.06271 [gr-qc]].

[39] R. Dey, S. Liberati and D. Pranzetti, "Spacetime thermodynamics in the presence of torsion," Phys. Rev. D 96 (2017) no.12, 124032 arXiv:1709.04031 [gr-qc]]. 